\title{
Characteristics of Colon Crypt Stem Cells in Preserved Epithelial Cell Clumps in Dextran Sulfate Sodium-induced Mouse Model of Ulcerative Colitis
}

\section{Mio Kobayashi}

Tokyo University of Agriculture and Technology

Risako Yamashita

Tokyo University of Agriculture and Technology

Ryo Ichikawa

Tokyo University of Agriculture and Technology

Makoto Shibutani

Tokyo University of Agriculture and Technology

Toshinori Yoshida ( $\nabla$ yoshida7@cc.tuat.ac.jp)

Tokyo University of Agriculture and Technology

\section{Research Article}

Keywords: Bmi1, DII1, Lgr5, epithelial clumps, regeneration

Posted Date: January 15th, 2021

DOl: https://doi.org/10.21203/rs.3.rs-144494/v1

License: (9) This work is licensed under a Creative Commons Attribution 4.0 International License. Read Full License 


\section{Abstract}

Crypt stem cells rescue the colorectum from refractory ulcers in ulcerative colitis (UC). We previously reported that clumps of a few epithelial cells were scattered in ulcer regions in a dextran sulfate sodium (DSS)-induced mouse model of UC; however, the origin of the clumps is unknown. We determined the immunohistochemical expression of stem-cell markers in the epithelial clumps in female Balb/c mice administered DSS in drinking water for 6 days, followed by withdrawal of DSS for 6 days. Similar to the characteristics of UC, the ulcers were more severe in the distal region close to the anus than in the proximal region of the colorectum. Quantitative analyses revealed that the epithelial clumps appeared in relation to the severity of ulcer, and they expressed the cell adhesion molecules E-cadherin and $\beta$-catenin. Among stem cell markers, the epithelial clumps primarily expressed +5 cell marker Dll1, followed by +4 cell marker Bmi1 and crypt stem cell marker Lgr5 in that order. Nuclear expression of Sox9, but not Ki-67 and $\beta$-catenin was identified in the clumps. The present results suggest that the epithelial clumps comprised crypt-derived stem cells with the potential to regenerate crypts, which could serve as a potential treatment strategy for UC.

\section{Introduction}

Epidemiological analyses raise concerns regarding the increased incidence of patients with inflammatory bowel disease (IBD) worldwide; the incidence is estimated to have increased more than 2-fold in selected industrialized countries of the Western world between 2010 and 2030, thus representing global IBD burden ${ }^{1}$. Ulcerative colitis (UC) is an IBD, characterized by inflammation and ulceration of the mucosa limited to the colorectum in patients with repeated relapse and remission; it leads to adverse effects on the quality of life of the patients and the risk of colon cancer ${ }^{2,3}$. The disease activity of UC is assessed by clinical, biochemical, endoscopic, or histologic analyses, and remission at each level has been discussed to achieve therapeutic goals, that is, mucosal healing ${ }^{4}$. Histological remission has recently been recognized as an ultimate indicator of mucosal healing in $\mathrm{UC}^{5-7}$. To accomplish this, intestinal crypt stem cells play a central role in crypt regeneration, which is anticipated to be a potential therapeutic strategy for patients with $U^{8}$.

An animal model using dextran sulfate sodium (DSS)-treated mice have been widely accepted for analyzing ulcers and subsequent regeneration in a time-dependent manner and for determining histological healing ${ }^{9}$. Initial mucosal damage may be immediately rescued by restitution, which is the migration of monolayered epithelial cells from the surrounding mucosa reserved at the ulcer edge to cover the denuded $a \mathrm{area}^{10}$. We and other researchers reported a monolayer of restituted epithelium covering the surface of ulcer areas during the recovery period after DSS administration in mice ${ }^{11-14}$. Together with the observation of restitution, we reported that small cell clumps remained under the lamina propria after induction of ulcers in DSS-treated mice ${ }^{11,12}$. The epithelial clumps appeared alongside or before the period of restitution. These clumps consisted of a few epithelial cells; immunohistochemical studies demonstrated that they expressed cytokeratin AE1/AE3 and lacked the 
mesenchymal marker vimentin; cell proliferation markers proliferating cell nuclear antigen (PCNA) and 5bromo-2'-deoxyuridine (BrdU); and mucin, as indicated by PAS reaction ${ }^{11}$. However, the origin of the epithelial clumps is unknown.

Mucosal healing is regulated by stem cell lineages in the crypt of the small and large intestines: activecycling/proliferating intestinal stem cells (aISC) or crypt base columnar (CBC) cells and quiescent/slowcycling reserve ISC (rISC) or label-retaining cells (LRC) ${ }^{15}$. aISC/CBC cells expressing leucine-rich repeatcontaining G-protein coupled receptor 5 (Lgr5) are essential for the maintenance of mucosal homeostasis; they reside in the crypt bottom as self-renewing stem cells generating daughter progenitors, which differentiate into absorptive and secretory lineages ${ }^{16-18}$. The cells that reside in the fourth position and the fifth to fifteenth positions from the crypt bottom are known as +4 and +5 cells, respectively, and are characterized by the expression of the progenitor cell markers $B$ lymphoma Mo-MLV insertion region 1 homolog (Bmi1), and Notch ligands jagged1, delta-like1 (DII1), respectively. These cells are categorized as rISC; they can differentiate into functional absorptive and secretory epithelial cells and revert to stem cell lineages when aISC is lost or injured ${ }^{19,20}$. Sex-determining region $Y(S R Y)$-box transcription factor 9 (SOX9) is a negative regulator of the Wnt-beta-catenin pathway, which regulates stemness in aISC and $\mathrm{rISC}^{21,22}$. In this study, we hypothesized that the strayed epithelial clumps might be derived from inflammation-resistant stem cells or their daughter progenitor cells, which have the potential to regenerate crypts and mucosal healing in refractory ulcers of the colorectum in a DSS-induced UC model. To test this hypothesis, we conducted morphometric and immunohistochemical studies of the epithelial clumps in the proximal regions with slight-to-moderate ulcers and in the distal regions close to the anus with more severe ulcers in the colorectum and elucidated whether the epithelial clumps maintain the characteristics of Lgr5-expressing crypt stem cells or that of Bmi1-, Dlli1-, or Sox9-expressing daughter progenitor cells.

\section{Materials And Methods}

\section{Chemicals}

DSS (molecular weight 36,000-50,000, CAS number 9011-18-1) was purchased from MP Biomedicals (Santa Ana, CA, USA).

\section{Animals}

Four-week-old female BALB/cAnNCrlCrlj mice $(n=34$, Charles River Laboratories Japan, Inc., Kanagawa, Japan) were acclimated for 9 days to the testing environment under controlled temperature $\left(23 \pm 3^{\circ} \mathrm{C}\right)$, humidity $(50 \pm 20 \%)$, and light (12:12-h light-dark cycle) conditions. Three to four mice were maintained in an individual plastic cage with enrichment and safe harbor and provided free access to a basal diet (Oriental MF, Oriental Yeast, Tokyo, Japan) and tap water. Food consumption and water intake were measured twice a week per cage, and body weight was measured daily during the study. All procedures in this study were conducted in accordance with the Guidelines for Proper Conduct of Animal Experiments (Science Council of Japan, June 1, 2006), and the protocols were approved by the Animal Care and Use 
Committee of the Tokyo University of Agriculture and Technology (Fuchu, Tokyo, Japan). The study was carried out in compliance with the ARRIVE guidelines.

\section{Experiment design of DSS-induced colitis}

Animals were divided into two groups according to body weight at the initiation of the study: the negative control group $(n=4)$ and DSS group $(n=30)$. Negative controls (untreated mice) received sterile distilled water. In the DSS-treated group, the mice received 5\% (w/v) DSS dissolved in sterile distilled water as drinking water for 6 days, from Day 1 to Day 6 , followed by withdrawal of DSS for 6 days for induction of colitis. The DSS solution was prepared twice during the administration. The DSS dose was selected on the basis of a preliminary study. The mice were anesthetized on Day 6 (control, $n=2$; DSS-treated, $n=11$ ) and Day 12 (control, $n=2$; DSS-treated, $n=10$ ) using isoflurane inhalation, euthanized via exsanguination, and subsequently necropsied. The other mice showed moribundity and lethality during the study, following which they were necropsied.

\section{Assessment of Disease Activity Index of Colitis}

Each mouse was evaluated daily for diarrhea, fecal blood (Uropaper® III Eiken, Eiken Chemical Co., Inc., Tokyo, Japan), and body weight loss for the sake of being used as the disease activity index (DAI), as previously described $^{11,23}$ (Supplemental Table 1). Fecal blood was scored by cutting the collected feces in half, putting them in a PCR tube containing physiological saline for 30 seconds, and subsequently dipping a Uropaper ${ }^{\circledR}$ III Eiken in the tube for another 1 minute. DAI was calculated by summing the scores for diarrhea, fecal blood, and body weight loss.

\section{Tissue Preparation and Histological Evaluation of Colitis}

The colorectum was removed from the large intestine and measured for length ${ }^{12}$. The colorectum was divided into two segments, distal and proximal regions, and fixed in $4 \%$ paraformaldehyde in phosphate buffer solution (PFA). The fixed colorectum was routinely embedded in paraffin, sectioned, and subjected to hematoxylin and eosin (H\&E) staining. Histological evaluation was performed by scoring the extent of tissue injury based on inflammation, mucosal loss, and edema using H\&E-stained specimens, as previously described $^{12}$ (Supplemental Table 2). Epithelial clumps at the lamina propria were observed as previously reported ${ }^{11}$ and classified into seven types based on morphological characteristics. The clumps were subclassified into type 1 to 4 , according to the number of nuclei: type 1 exhibited a single nucleus; type 2, two or three nuclei; type 3, four to nine nuclei; and type 4, 10 nuclei or more (Supplemental Fig. 1). These clumps were additionally subclassified into types a and b, according to the presence of luminal structure: type a exhibited luminal structure, while type b lacked luminal structure. By combining types a and b, and types 1 to 4, there were 7 subtypes of epithelial clumps, except for type 4b. Furthermore, the number of epithelial clumps was evaluated by counting the number of clumps per millimeter of the total mucosal length on H\&E sections using a vertical slide system (VS120, Olympus Corporation, Tokyo, Japan).

\section{Immunohistochemical analysis}


Immunostaining was performed manually using antibodies against LGR5, SOX9, alpha smooth muscle actin (a-SMA), $\beta$-catenin, BMI1, DLL1, myeloperoxidase, ionized calcium binding adapter protein 1 (Iba1), E-cadherin, and Ki-67 (Supplemental Table 3). The primary antibodies were applied overnight at $4{ }^{\circ} \mathrm{C}$. An avidin-biotin-peroxidase complex method was employed using the VECTASTAIN ${ }^{\circ}$ Elite ABC kit (Vector Laboratories Inc., Burlingame, CA, USA) with 3,3'-diaminobenzidine as a chromogen, followed by counterstaining with hematoxylin for microscopic examination. The epithelial cell clumps expressing LGR5, SOX9, BMI1, DLL1, $\beta$-catenin, or E-cadherin were quantitatively evaluated using a vertical slide system (VS120, Olympus Corporation). The number of epithelial clumps with low or high immunopositive reactions were counted per unit of mucosal length $(\mathrm{mm})$.

\section{Double immunohistochemical staining}

Double immunohistochemical staining was conducted as previously reported ${ }^{24}$ using SOX9 and DLL1 as the primary antibodies. In brief, deparaffinization was performed as described above, and immunopositive reactions were visualized using the VECTASTAIN $®$ Elite ABC kit and 3,3'diaminobenzidine. The sections were treated with $0.1 \mathrm{M}$ glycine hydrochloride buffer twice for 25 minutes, followed by overnight incubation with the primary antibodies at $4{ }^{\circ} \mathrm{C}$. An avidin-biotin-alkaline phosphatase complex method was followed using the VECTASTAIN® ABC-AP kit with an alkaline phosphatase substrate as a chromogen; Vector®Blue (Vector Laboratories Inc.), followed by using a water-soluble encapsulant.

\section{Statistical analysis}

Multiple comparison test, Tukey-Kramer test, or Steel-Dwass test was used to analyze the body weight, the length of the colorectum, and the number of epithelial cell clumps on the H\&E-stained and immunostained sections. The length of the colorectum was statistically analyzed using Student's unpaired t-test or the Aspin-Welch test. Histological evaluation (inflammation, mucosal loss, and edema) was statistically analyzed using the Wilcoxon signed-rank test. All data are represented as the mean and standard deviation (SD), except for the DAl data.

\section{Results}

\section{DSS administration and subsequent withdrawal exacerbates disease activity index and shortens colon length}

The body weight of the DSS-treated mice began to decrease on and after Day 6 and continued to decrease until the end of the study (Fig. 1A). The scores for diarrhea and fecal blood in the DSS-treated mice began to increase on and after Day 3 (Fig. 1B, C). The diarrhea score continued to increase until Day 8 ; the score for fecal blood peaked on Day 6 and decreased thereafter. DAl, as determined by summing the scores of these three parameters, peaked on Day 6 and decreased on and after Day 9 (Fig. 1D). Shortening of the colon length is a reliable indicator of colitis ${ }^{12}$. Macroscopically, the mean colorectal lengths in the control mice were 9.3 and $11.3 \mathrm{~cm}(\mathrm{n}=2$ each) on Day 6 and Day 12, respectively, whereas 
those in the DSS-treated mice were 6.4 and $7.2 \mathrm{~cm}$ on Day $6(n=11)$ and Day $12(n=10)$, respectively (Fig. 2A). Clinical and macroscopic quantifications suggested that DSS challenge induced colitis-related illness and macroscopic changes, as previously reported ${ }^{12}$.

\section{DSS administration and subsequent withdrawal induces more severe colitis in the distal colorectum}

Colitis was estimated by scoring inflammation, edema, and mucosal loss, and the scores were compared on Day 6 and Day 12 or the distal region close to the anus with the proximal region of the colorectum. In the distal region, the scores of inflammation and mucosal loss were almost the same between Day 6 and Day 12 (Fig. 2B-D), as the lesions were already severe even on Day 6. In the proximal region, the score of each parameter was significantly higher on Day 12 than on Day 6 , as the lesions progressed in a timedependent manner. The score of each parameter was significantly higher in the distal region than in the proximal region on Day 6 and Day 12. Inflammation and edema were sought to be better indicators than edema in the present study.

\section{Clumps of epithelial cells appear in ulcer region in the colorectum}

We quantitatively examined the epithelial clumps in the lamina propria of the ulcer site in both the distal and proximal regions of the colorectum (Fig. 3A). In the DSS group, the number of clumps increased on Day 12 in both the distal and proximal regions when compared with that on Day 6; there was a significant difference between the values on Day 6 and Day 12 in the proximal region (Fig. 3B). The number of clumps was significantly higher in the distal region than in the proximal region on Day 6 ; this parameter was associated with the severity of colitis.

\section{Clumps of epithelial cells express markers of crypt stem cells and daughter progenitor cells}

We first confirmed that the epithelial cells in the normal crypts, remaining crypts, and regeneration crypts expressed the epithelial cell adhesion markers E-cadherin and $\beta$-catenin and the stem cell/daughter progenitor cell markers LGR5, BMI1, DLL1, and SOX9 (Supplemental Fig. 2). The epithelial clumps expressed these cell markers; E-cadherin and $\beta$-catenin were restricted to the cell-cell border, and LGR5 and DLL1 were observed in the cytoplasm, while SOX9 and BMI1 were detected in the nucleus (Fig. 4A-C, $5 A-C)$. We observed that the expression of each marker varied among the epithelial clumps; therefore, we counted each clump with negative staining or positive staining at a low or high level per unit of length. Ecadherin $^{\text {low }}$ or $\beta$-catenin ${ }^{\text {low }}$ clumps were a major population, while E-cadherin ${ }^{\text {high }}$ or $\beta$-catenin ${ }^{\text {high }}$ clumps were a minor population (Fig. 4D, E, G, H). The number of E-cadherin ${ }^{\text {low }}$ or $\beta$-catenin ${ }^{\text {low }}$ clumps were significantly increased in the proximal region on Day 12 compared with the values on Day 6 , as the disease progressed. SOX $9^{\text {low }}$ clumps were more abundant in both the distal and proximal regions on Day 6 than on Day 12, while the number of SOX $9^{\text {negative }}$ clumps were significantly increased on Day 12 compared with that on Day 6 (Fig. 4F, I). Lgr5 ${ }^{\text {low }}$ clumps were identified as the most minor population in 
both the distal and proximal regions on Day 6 and Day 12 (Fig. 5D, G). BMI1 ${ }^{\text {low }}$ clumps were identified in both the distal and proximal regions on Day 6 and Day 12, and BMI1 ${ }^{\text {high }}$ clumps were detected in the distal region on Day 12 (Fig. 5E, H). DLL1 $1^{\text {high }}$ and DLL1 $1^{\text {low }}$ clumps appeared to be more in number than LGR5 ${ }^{\text {low }}$ or BMI1 ${ }^{\text {low }}$ clumps on Day 6 and Day 12 (Fig. 5F, I). The clumps did not express the myofibroblast marker aSMA, macrophage marker Iba1, or cell proliferation marker Ki-67.

To determine the coexpression of SOX and DLL1 in the clumps, we confirmed the expression of these markers in normal crypts, residual crypts, and regenerative crypts (Supplemental Fig. 3). SOX9/DLL1coexpressing clumps were evident in the distal region on Day 6 (Fig. 6A-C). DLL1-single expressing clumps were significantly increased in the distal region on Day 12, compared with the SOX9/DLL1-

coexpressing cells. The percentage of SOX9 ${ }^{\text {low }}$ DLL1 $1^{\text {low }}$ or SOX9 $9^{\text {high }} \mathrm{DLL} 1^{\text {low }}$ clumps was higher than that of SOX9high DIl1 ${ }^{\text {high }}$ or SOX9low DIl1 ${ }^{\text {high }}$ clumps (Fig. 6D).

\section{Discussion}

The present study revealed four major findings on preserved epithelial clumps in a mouse model of UC. First, the epithelial clumps were detected in the lamina propria in the ulcer region in H\&E-stained sections with easy microscopic identification for pathologists; they can be classified into four subgroups according to various morphological characteristics in terms of the number of nuclei and intracellular space in the cytoplasm or intercellular crypt-like structure (Supplemental Fig. 1). Second, most epithelial clumps expressed markers for the epithelial cell adhesion molecules E-cadherin (approximately 80\%) and $\beta$-catenin (approximately 60\%) (Fig. 4G, H). Third, the epithelial clumps heterogeneously expressed the alSC marker Lgr5 and rISC markers Bmi1 and DII1; a major population of the epithelial clumps expressed DII1 (50-80\%), a part of the population expressed Bmi1 (approximately $20 \%$ or less), and a minor population expressed Lgr5 (Fig. 5G-I). Fourth, a population of epithelial clumps expressed the stem cell niche marker Sox9; however, its expression was notably reduced with disease progression (approximately $50 \%$ on Day 6 and a low percentage on Day 12) (Fig. 4I). Furthermore, the coexpression profiles of Sox 9 and DLLI indicated that a major population of the epithelial clumps was DII1-single-positive clumps, and a minor population was SOX9 ${ }^{\text {low }}$ DLL1 $1^{\text {low }}$ or SOX9 ${ }^{\text {high }}$ DLL1 ${ }^{\text {low }}$ clumps (Fig. 6C, D). The results of the present study suggest that the epithelial clumps were derived from the crypt base in the villous-crypt axis; the heterogenous cellular properties of the stem cells or their daughter cells in these clumps might be dependent on the degree of resistance to inflammation-mediated epithelial loss.

The intestinal epithelial crypt has a capacity of constant renewal by proliferating stem cells owing to maintenance of homeostasis ${ }^{25}$. Lgr5 ${ }^{+}$aISC rapidly proliferates for self-renewal and the generation of all functional epithelial lineages under normal conditions ${ }^{15,26}$. We detected a small number of Lgr $5^{+}$ epithelial clumps in the ulcer region; these results might be supported by the evidence that Lgr5 ${ }^{+}$ aISC/CBC cells in the colon crypt are particularly susceptible to mucosal damage in mice with DSSinduced colitis ${ }^{27}$ and radiation-exposed mice ${ }^{28}$. The Lgr $^{\text {high }} \mathrm{Ki}^{-67^{+}} \mathrm{ISC} / \mathrm{CBC}$ cells in the small intestine are active with rapid cell cycling for controlling self-renewal and producing progenitor cells; in contrast, 
the colon $\mathrm{Lgr} 5^{\text {low }} \mathrm{Ki}-67^{-}$ISC/CBC cells are quiescent with the potential to retain epithelial self-renewal over long periods of time ${ }^{29,30}$. The negative nuclear expression of $\mathrm{Ki}-67$ in the epithelial clumps might be consistent with the presence of quiescent $\mathrm{Lgr}^{+}$ISC/CBC cells in the colon crypt. The epithelial clumps were classified as Lgr $5^{\text {high }}$ or Lgr5 ${ }^{\text {low; }}$; the percentage of $L g r 5^{\text {low }}$ epithelial clumps was higher than that of the Lgr5 ${ }^{\text {high }}$ epithelial clumps (Fig. 5D, G). Several researchers have reported that $\mathrm{Lgr}^{\text {low }} \mathrm{Ki}^{-67^{-}}$ISC/CBC cells generate enteroendocrine cells, goblet cells, tuft cells, and deep crypt secretory (DCS) cells and constitute ISC/CBC cells by self-renewal ${ }^{20,31-33}$. Lgr5 is directly regulated by Wnt signaling: Lgr5 is targeted to the $\beta$-catenin complex, committing to nuclear-translocated $\beta$-catenin ${ }^{34}$. Lgr $5^{\text {high }} \beta$ catenin ${ }^{\text {nucleart }}$ cells may lead to premalignant conditions caused by the activation of Wnt signaling ${ }^{35}$; however, no nuclear translocation of $\beta$-catenin was identified in the epithelial clumps. Thus, Lgr5 ${ }^{\text {low }} \mathrm{Ki}-67^{-}$ $\beta$-catenin ${ }^{\text {nuclear- }}$ epithelial clumps were assumed to be quiescent for the sake of potentially regenerating the crypt and healing the mucosa over long periods of time.

DII $1^{\text {high }}$ cells are called +5 cells and are categorized as rISC or label-retaining cells (LRC) with quiescent or slow-cycling status; however, after aISC/CBC cell are damaged, the cells can quickly differentiate into secretory lineage cells, that is, tuft cells, enteroendocrine cells, goblet cells, and Paneth cells ${ }^{20}$. The DCS cells in the colon express the Notch ligand DII1 and likely serve as a niche for stem cells to regulate their homeostasis ${ }^{36}$. In addition, transit amplifying (TA) cells are rapidly cycling DII1+ ${ }^{+} \mathrm{Ki}-67^{ \pm}$daughter progenitor cells; they undergo differentiation into an absorptive lineage, that is, enterocytes ${ }^{33,37}$. Considering that DII1 ${ }^{+}$epithelial clumps were the most common type of epithelial clumps according to the present study, we therefore propose that DII ${ }^{\text {high }}$ epithelial clumps might maintain a potential for reviving stemness, followed by mucosal healing in the ulcer region.

Sox9 belongs to the Sox family of transcription factors, and it is required in the small intestine for the differentiation of the secretory lineage and proliferation of $\mathrm{rISC}^{25}$. Additionally, Sox9 plays a role in negative feedback with respect to the activity of the Wnt- $\beta$-catenin pathway; the overexpression of Sox 9 inhibits the Wnt- $\beta$-catenin pathway, which promotes tumorigenesis, while appropriate expression of Sox 9 is required for Wnt- $\beta$-catenin pathway activity in $\mathrm{rISC}^{21}$. Roche et $\mathrm{al}^{22}$ proposed that Sox 9 expression levels were categorized as high or low according to intensity in ISC: Sox $9^{\text {high }}$ rISC is quiescent and slowcycling under a homeostatic state; however, it can be converted to Sox $9^{\text {low }}$ aISC, facilitating epithelial repair, when aISC is lost after damage. Although the role of Sox9 in the colon is not fully understood at present, it is proposed that Sox9 is committed to morphogenesis in the colon epithelium and that it plays a role in the differentiation of the secretory lineage, especially goblet cells ${ }^{38,39}$. Coexpression analyses of Sox9 and markers of aISC or rISC would confirm the potential of restoring the colon crypt. Although the coexpression analysis of Sox9 and Dlli has not been determined in normal and injured colon crypts to our knowledge, we speculated that SOX $9^{\text {low }}$ DLL $1^{\text {high }}$ clumps might have the potential to restore the crypts in ulcer sites. 
Bmi $1^{+}$quiescent ISC (qISC)/LRC or +4 cells play a crucial role in facilitating regeneration in injured crypts, indicating that they compensate for the absence of $\mathrm{Lgr}^{+}$cells in the small intestine ${ }^{28,40}$; however, they tend to be insensitive to the absence of $\mathrm{Lgr}^{+}$cells in the distal small intestine and $\operatorname{colon}^{40}$. Sox 9 directly binds to the promoter of $\mathrm{Bmi}$, thus regulating the gene expression of $\mathrm{Bmi}{ }^{41}$. Bmi1 is an epigenetic regulator, which restricts cell proliferation and shows a strong correlation with Sox 9 expression ${ }^{42}$. As damage-sensitive SOX9+ clumps markedly reduced between Day 6 and Day 12, Bmi ${ }^{+}$ clumps might exhibit a low potential for mucosal healing in the DSS-induced UC model.

Considering the above results, we propose a model for the formation of epithelial clumps in the colorectum in a DSS-induced mouse model. Inflammatory stimuli impair $\mathrm{Lgr}^{+}$alSC/CBC cells and $B m i 1^{+}$qISC/LRC (or +4 cells), as these cells are more sensitive than DII $1^{+}$rISC (or TA/ +5 cells), which are relatively resistant to inflammatory stimuli and which can survive as small clumps of epithelial cells in the ulcer region. Furthermore, the colon crypt base contains Sox9 ${ }^{+}$DCS cells, instead of Paneth cells; DCS cell-derived clumps can be preserved at an early phase of colitis and subsequently impaired by longlasting inflammation. The present study indicated that clumps of epithelial cells heterogeneously expressed markers of aISC/CBC cells and their daughter rISC in the colon crypt. The results suggested that the DII1+ crypt-derived clumps were resistant to DSS-induced colitis; they might have retained the potential to regenerate the crypt and heal the mucosa. Transplantation of primary crypt stem cells and induced human pluripotent stem cells to the colorectum might enhance mucosal healing in patients with UC; however, this approach has some limitations, including interruption with gut microflora and transplant rejection by the immune system ${ }^{8}$. Induction of regenerative crypts from epithelial clumps with stem cell potential would be an alternative experimental approach to stem cell therapy; therefore, further study is required to observe the morphological changes in epithelial clumps and the relationship between the clumps and regenerative crypts through the process of mucosal healing in the DSS-induced UC model.

\section{Declarations}

\section{Acknowledgement}

The study is supported by the TAMAGO (Technologically Advanced Research through Marriage of Agriculture and Engineering as Groundbreaking Organization) grant from the Tokyo University of Agriculture and Technology (Tokyo, Japan).

\section{Author contributions}

M.K. and T.Y. conceived the project, designed, and conducted animal experiments, analyzed and interpreted the data, prepared figures and tables, and wrote the main manuscript text. R.Y. and R.I. helped in animal experiments and immunohistochemistry. M.S. initiated the project and interpreted the results. All authors reviewed the manuscript. 


\section{Competing interest statement}

The authors declare that they have no competing interests.

\section{References}

1. Kaplan, G.G. \& Windsor, J.W. The four epidemiological stages in the global evolution of inflammatory bowel disease. Nat Rev Gastroenterol Hepatol 18, 56-66 (2021).

2. Kaur, A. \& Goggolidou, P. Ulcerative colitis: understanding its cellular pathology could provide insights into novel therapies. J Inflamm (Lond) 17, 15 (2020).

3. Porter, R.J., Kalla, R. \& Ho, G.T. Ulcerative colitis: Recent advances in the understanding of disease pathogenesis. F1000Res. 9, F1000 Faculty Rev-294 (2020).

4. Rogler, G., Vavricka, S., Schoepfer, A. \& Lakatos, P.L. Mucosal healing and deep remission: What does it mean? World J Gastroentero/ 19, 7552-7560 (2013).

5. Kanazawa, M. et al. Relationship between endoscopic mucosal healing and histologic inflammation during remission maintenance phase in ulcerative colitis: a retrospective study. Endosc Int Open 7, E568-E575 (2019).

6. Lobatón, T. et al. Prognostic value of histological activity in patients with ulcerative colitis in deep remission: A prospective multicenter study. United European Gastroenterol J 6, 765-772 (2018).

7. Magro, F. et al. ECCO Position Paper: Harmonisation of the approach to Ulcerative Colitis Histopathology. J Crohns Colitis jjaa110 (2020).

8. Holmberg, F.E.O. et al. Intestinal barrier integrity and inflammatory bowel disease: Stem cell-based approaches to regenerate the barrier. J Tissue Eng Regen Med 12, 923-935 (2018).

9. Randhawa, P.K., Singh, K., Singh, N. \& Jaggi, A.S. A review on chemical-induced inflammatory bowel disease models in rodents. Korean J Physiol Pharmacol 18, 279-288 (2014).

10. Liu, Y. \& Chen, Y.G. Intestinal epithelial plasticity and regeneration via cell dedifferentiation. Cell Regen 9, 14 (2020).

11. Kangawa, $\mathrm{Y}$ et al. Expression of A-kinase anchor protein 13 and Rho-associated coiled-coil containing protein kinase in restituted and regenerated mucosal epithelial cells following mucosal injury and colorectal cancer cells in mouse models. Exp Toxicol Pathol 69, 443-450 (2017b).

12. Kangawa, Y. et al. Suppression of epithelial restitution using an inhibitor against Rho-associated coiled-coil containing protein kinase aggravates colitis through reduced epithelial expression of Akinase anchor protein 13. Exp Toxicol Pathol 45, 493-505 (2017a).

13. Lopetuso, L.R. et al. IL-33 promotes recovery from acute colitis by inducing miR20 to stimulate epithelial restitution and repair. Proc Natl Acad Sci USA 115, E9362E9370 (2018).

14. Zimmerman, N.P., Kumar, S.N., Turner, J.R. \& Dwinell, M.B. Cyclic AMP dysregulates intestinal epithelial cell restitution through PKA and RhoA. Inflamm Bowel Dis 18, 1081-1091 (2012). 
15. Bankaitis, E.D., Ha, A., Kuo, C.J. \& Magness, S.T. Reserve stem cells in intestinal homeostasis and injury. Gastroenterology 155, 1348-1361 (2018).

16. Sato, T. et al. Paneth cells constitute the niche for Lgr5 stem cells in intestinal crypts. Nature 469, 415-418 (2011).

17. Liu, Y. \& Chen, Y.G. Intestinal epithelial plasticity and regeneration via cell dedifferentiation. Cell Regen 9:14 (2020).

18. Beumer, J. \& Clevers, H. Regulation and plasticity of intestinal stem cells during homeostasis and regeneration. Development 143, 3639-3649 (2016).

19. Pellegrinet, L. et al. DII1- and dll4-mediated notch signaling are required for homeostasis of intestinal stem cells. Gastroenterology 140, 1230-1240, e1-7 (2011).

20. van Es, J.H. et al. DII1+ secretory progenitor cells revert to stem cells upon crypt damage. Nat Cell Biol 14, 1099-1104 (2012).

21. Blache, P. et al. SOX9 is an intestine crypt transcription factor, is regulated by the Wnt pathway, and represses the CDX2 and MUC2 genes. J Cell Biol 166, 37-47 (2004).

22. Roche, K.C. et al. SOX9 maintains reserve stem cells and preserves radioresistance in mouse small intestine. Gastroenterology 149, 1553-1563, e10 (2015).

23. Sann H., Erichsen J., Hessmann M., Pahl A. \& Hoffmeyer A. Efficacy of drugs used in the treatment of IBD and combinations thereof in acute DSS-induced colitis in mice. Life Sci 92, 708-718 (2013).

24. Nakajima, K. et al. Developmental exposure to diacetoxyscirpenol reversibly disrupts hippocampal neurogenesis by inducing oxidative cellular injury and suppressed differentiation of granule cell lineages in mice. Food Chem Toxicol 136:111046 (2020).

25. Mori-Akiyama, Y. et al. SOX9 is required for the differentiation of paneth cells in the intestinal epithelium. Gastroenterology 133: 539-546 (2007).

26. Smith, N.R., Gallagher, A.C. \& Wong, M.H. Defining a stem cell hierarchy in the intestine: markers, caveats and controversies. J Physiol 594, 4781-4790 (2016).

27. Davidson, L.A. et al. Alteration of colonic stem cell gene signatures during the regenerative response to injury. Biochim Biophys Acta 1822, 1600-1607 (2012).

28. Yan, K.S. et al. The intestinal stem cell markers Bmi1 and Lgr5 identify two functionally distinct populations. Proc Natl Acad Sci USA 109, 466-471 (2012).

29. Barker, N. et al. Identification of stem cells in small intestine and colon by marker gene Lgr5. Nature 449, 1003-1007 (2007).

30. Snippert, H.J. et al. Intestinal crypt homeostasis results from neutral competition between symmetrically dividing Lgr5 stem cells. Cell 143, 134-144 (2010).

31. Sei, Y., Lu, X., Liou, A., Zhao, X., Wank, S.A. A stem cell marker-expressing subset of enteroendocrine cells resides at the crypt base in the small intestine. Am J Physiol Gastrointest Liver Physio/300, G345-G356 (2011). 
32. Buczacki, S.J.A. et al. Intestinal label-retaining cells are secretory precursors expressing Lgr5. Nature495, 65-69 (2013).

33. Basak, O. et al. Mapping early fate determination in Lgr5+ crypt stem cells using a novel Ki67-RFP allele. EMBO J 33, 2057-2068 (2014).

34. Deng, F. et al. YAP triggers the Wnt/ $\beta$-catenin signalling pathway and promotes enterocyte selfrenewal, regeneration and tumorigenesis after DSS-induced injury. Cell Death Dis 9, 153 (2018).

35. Zhou, X., Geng, L., Wang, D., Yi, H., Talmon, G. \& Wang J. R-Spondin1/LGR5 Activates TGF $\beta$ Signaling and Suppresses Colon Cancer Metastasis. Cancer Res 77, 6589-6602 (2017).

36. Sasaki, N. et al. Reg4+ deep crypt secretory cells function as epithelial niche for Lgr5+ stem cells in colon. Proc Natl Acad Sci USA 113, E5399-E5407 (2016).

37. Barker, N., van de Wetering, M. \& Clevers, H. The intestinal stem cell. Genes Dev 22, 1856-1864 (2008).

38. Bastide, P. et al. Sox9 regulates cell proliferation and is required for Paneth cell differentiation in the intestinal epithelium. J Cell Bio/ 178, 635-48 (2007).

39. Ramalingam, S., Daughtridge, G.W., Johnston, M.J., Gracz, A.D. \& Magness, S.T. Distinct levels of Sox9 expression mark colon epithelial stem cells that form colonoids in culture. Am J Physiol Gastrointest Liver Physio/ 302, G10-G20 (2012).

40. Tian, H. et al. A reserve stem cell population in small intestine renders Lgr5-positive cells dispensable. Nature 478:255-259 (2012).

41. Matheu, A. et al. Oncogenicity of the developmental transcription factor Sox9. Cancer Res 72, 13011315 (2012).

42. Aldaz, P. et al. SOX9 promotes tumor progression through the axis BMI1-p21CIP. Sci Rep 10, 357 (2020).

\section{Figures}



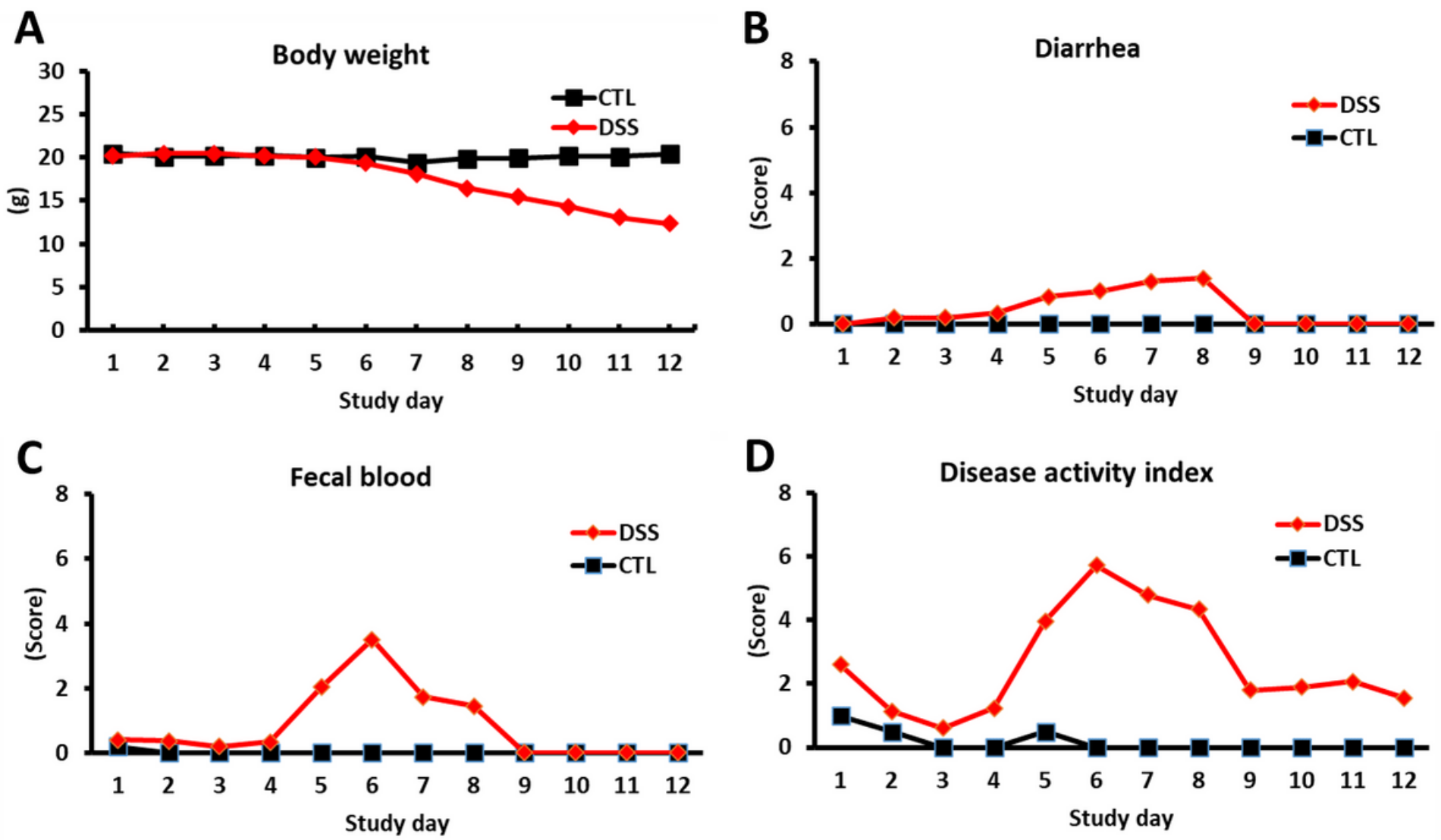

Figure 1

Disease activity index (DAI) in mice administered 5\% DSS in drinking water for 6 days, followed by withdrawal of DSS for 6 days. (A) Body weight (g). (B) Diarrhea (score). (C) Fecal blood (score). (D) DAl (score) is calculated by summing the scores for diarrhea, fecal blood, and body weight loss. The data represent the mean values for body weight, DAl, diarrhea scores, and fecal blood scores. $\mathrm{N}=2$, control group (CTL); N=30, DSS-treated group. 

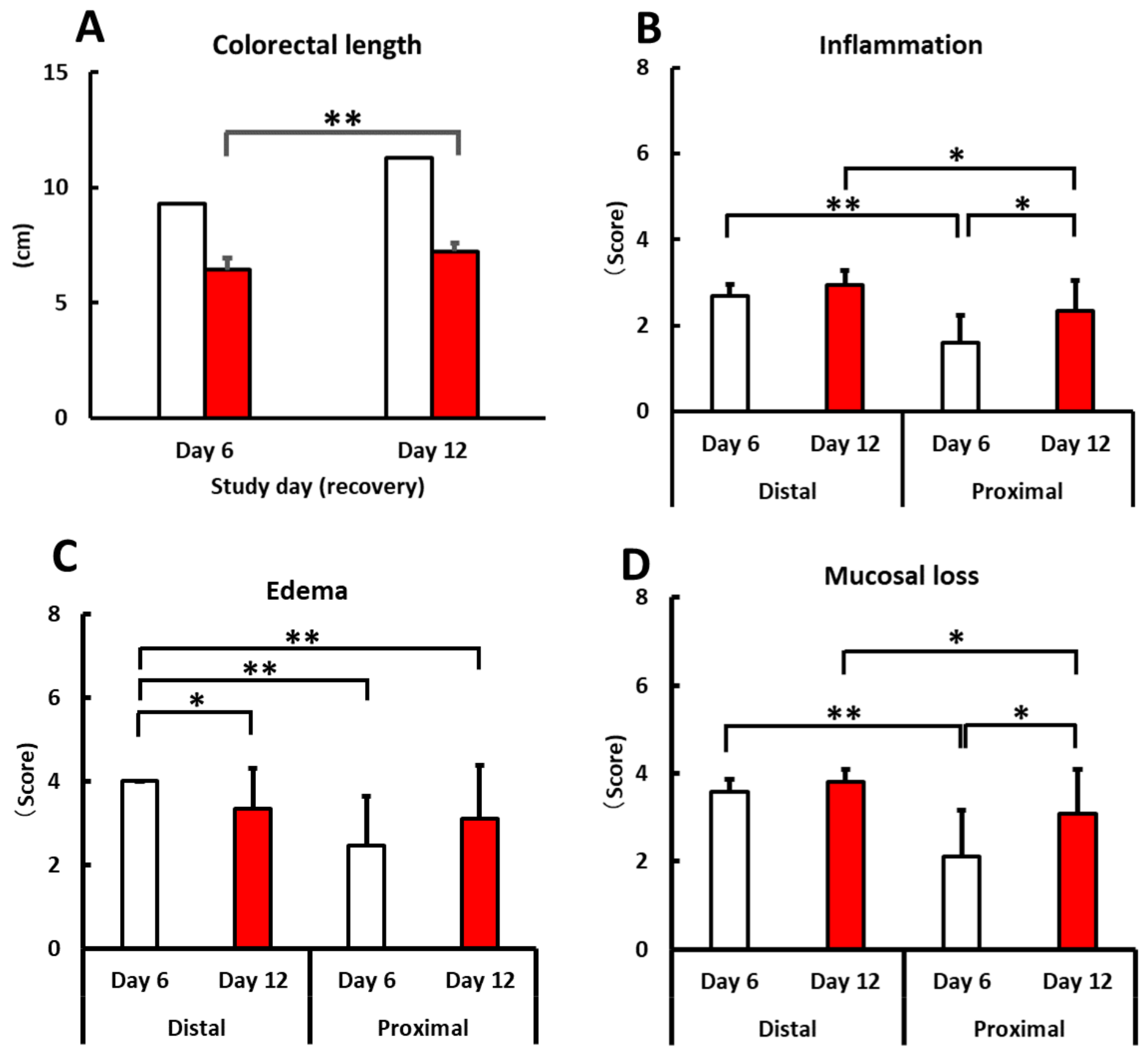

Figure 2

Colorectal length and histopathological scores in the colorectum in mice administered $5 \%$ DSS in drinking water for 6 days (Day 6), followed by withdrawal of DSS for 6 days (Day 12). (A) Colorectal length (cm). Scores for inflammation (B), edema (C), and mucosal loss (D) in the distal and proximal regions of the colorectum. The data represent the means and standard deviations. (A) **Significantly different compared with data from Day 6 ( $p<0.01$, Student's $t$ test or the Aspin-Welch test). (B-D) *, **Significantly different between each time point or region ( $p<0.05$ or 0.01 , the Mann-Whitney $U$ test). 
A

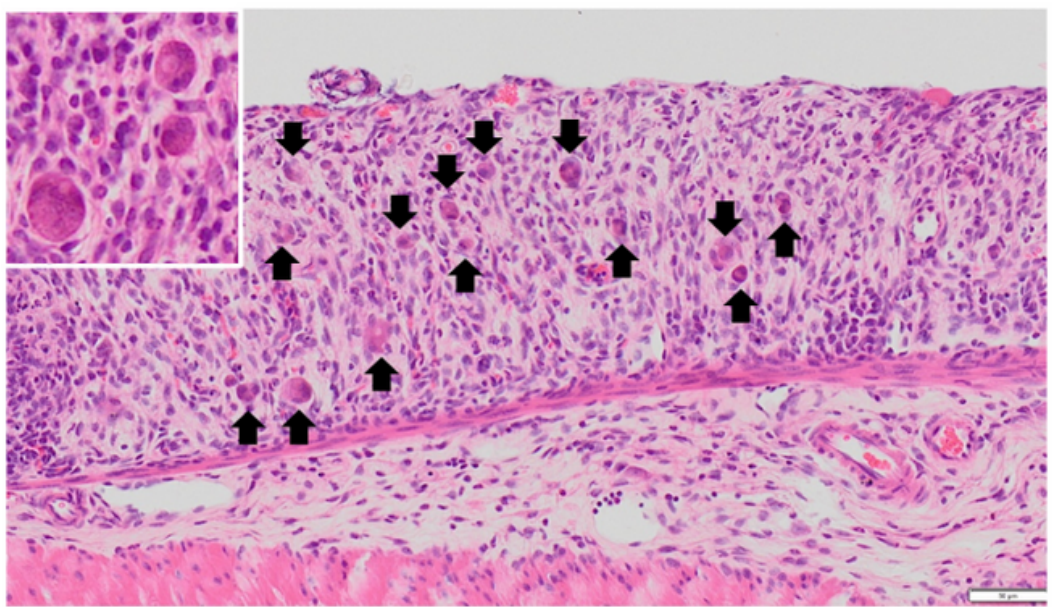

B

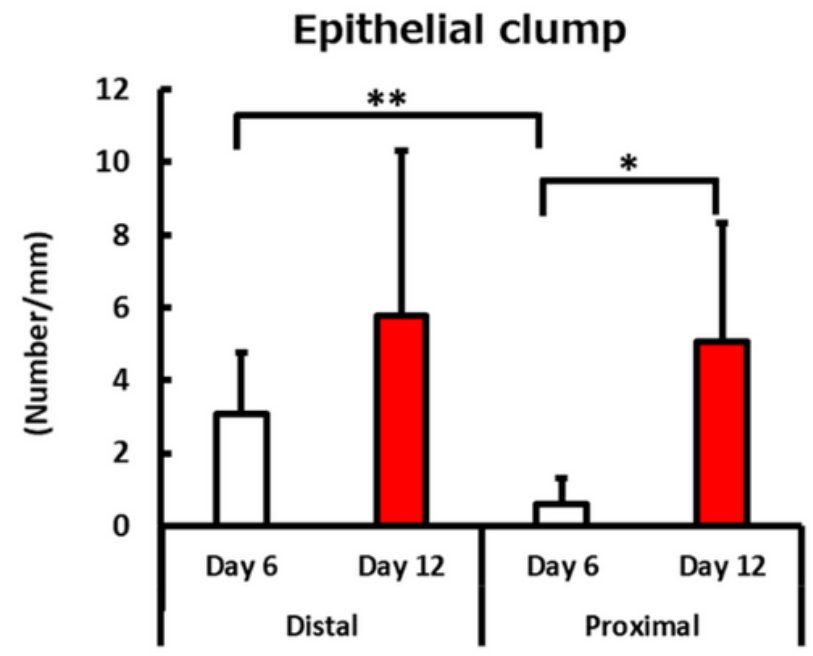

\section{Figure 3}

Representative images of epithelial clumps in the lamina propria in mice administered 5\% DSS in drinking water for 6 days (Day 6), followed by withdrawal of DSS for 6 days (Day 12). (A) Epithelial clumps are scattered in the lamina propria in the ulcer site (Arrows). Bar=50 $\mu \mathrm{m}$. Inset: a higher magnification of epithelial clumps. (B) Number of epithelial clumps per millimeter at the distal and proximal regions in the colorectum. The data represent the means and standard deviation. * ${ }^{\star} \star$ Significantly different between each time point or region ( $\mathrm{p}<0.05$ or 0.01 , the Tukey-Kramer test or Steel-Dwass test). 
A

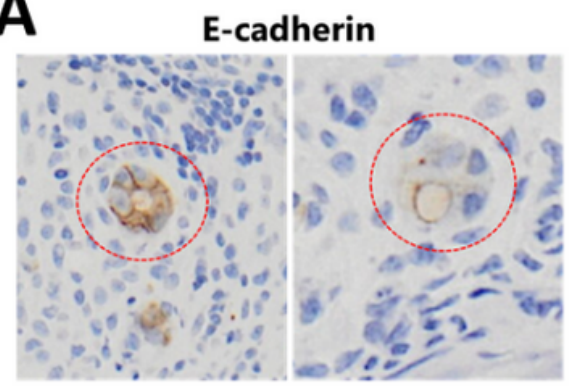

high

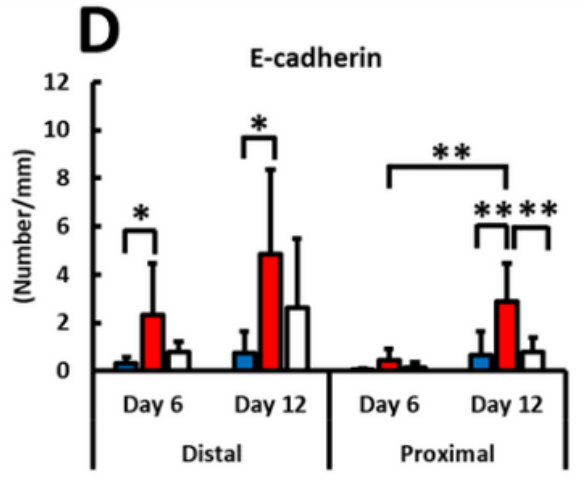

口high Dlow Dnegative

G

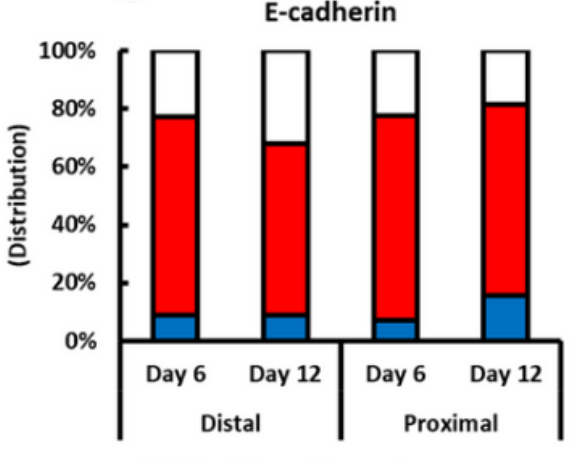

口high Dlow Dnegative

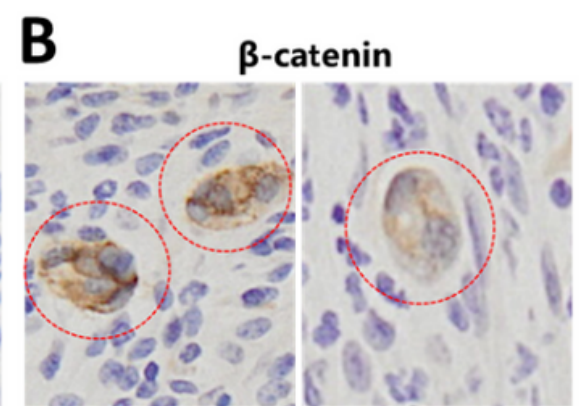

high

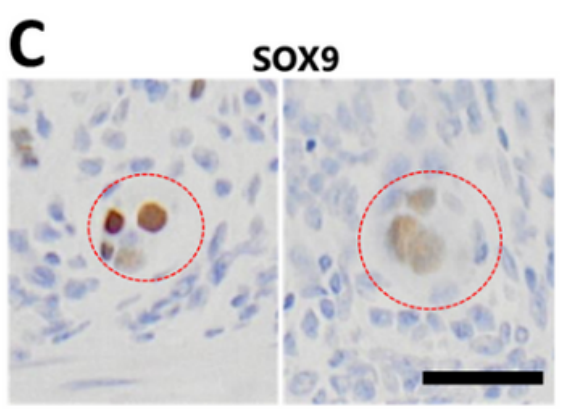

high low
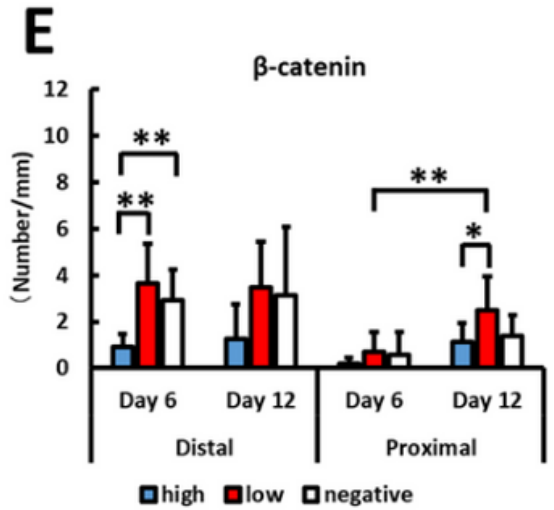

H

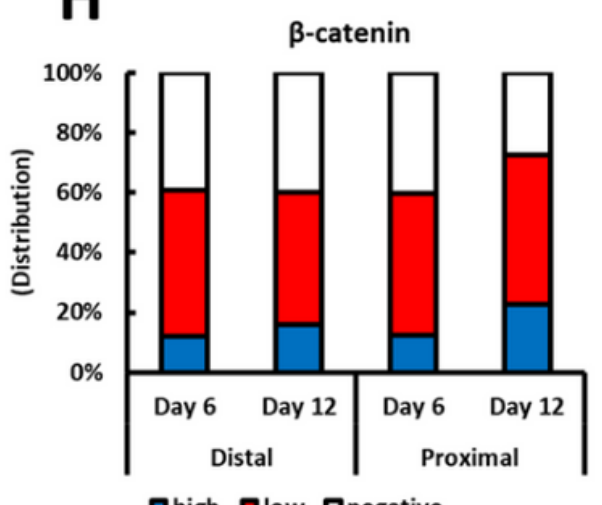

F

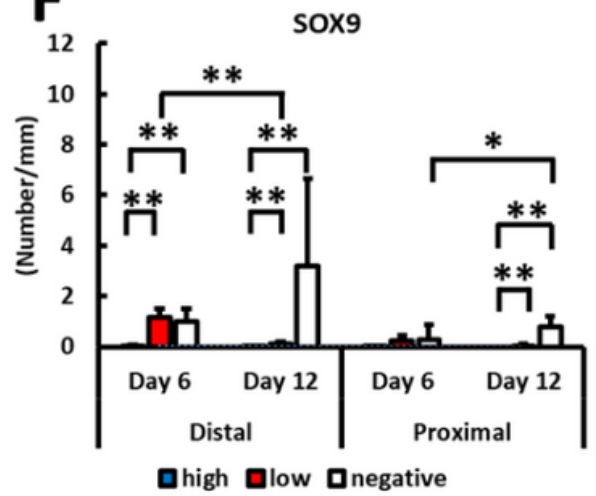

I

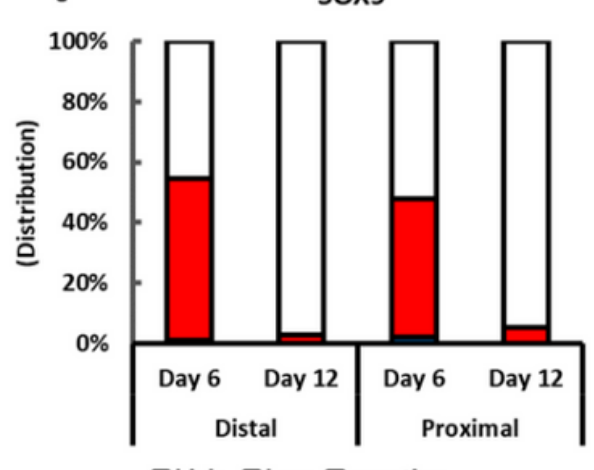

Figure 4

Coexpression analysis of cell adhesion and progenitor cell markers in the epithelial clumps in the lamina propria in the colorectum of mice administered 5\% DSS in drinking water for 6 days (Day 6), followed by withdrawal of DSS for 6 days (Day 12). Representative images of high and low expression of E-cadherin (A) and $\beta$-catenin (B) between cells and nuclear expression of SOX9 (C) in epithelial clumps (Red circles). Bar=10 $\mu \mathrm{m}$. The number of epithelial clumps with high and low expression levels (D-F) per millimeter and distribution (G-I) of each marker in the lamina propria in the distal and proximal colorectum. (D-F) The data represent the means and standard deviations. * ${ }^{*}$ Significantly different between each time point or region ( $p<0.05$ or 0.01 , the Tukey-Kramer test or Steel-Dwass test). 


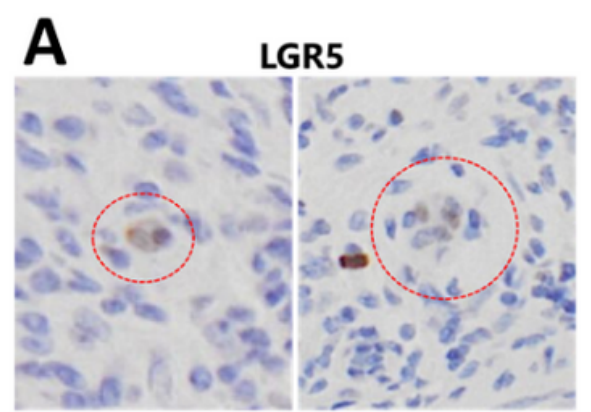

high
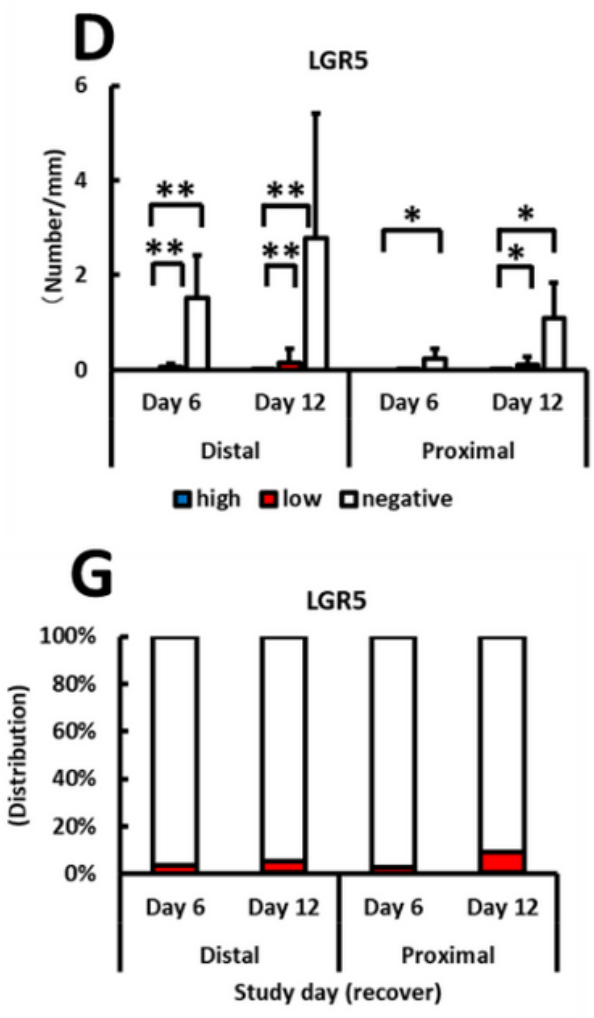

口high Dlow Dnegative

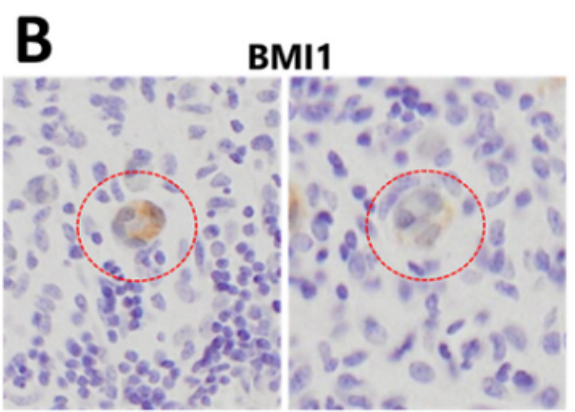

high

low

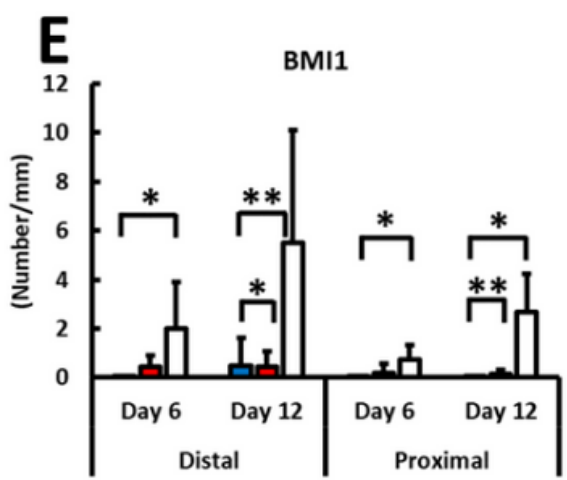

口high Dlow $\square$ negative

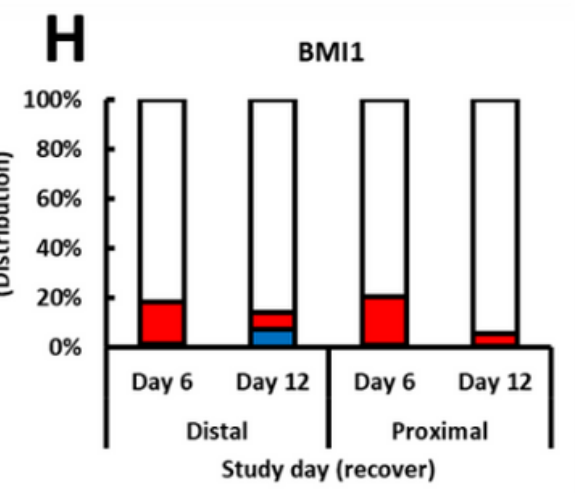

Qhigh Dlow Qnegative

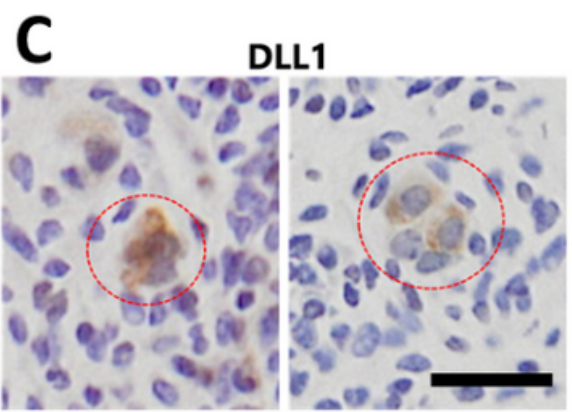

high

low
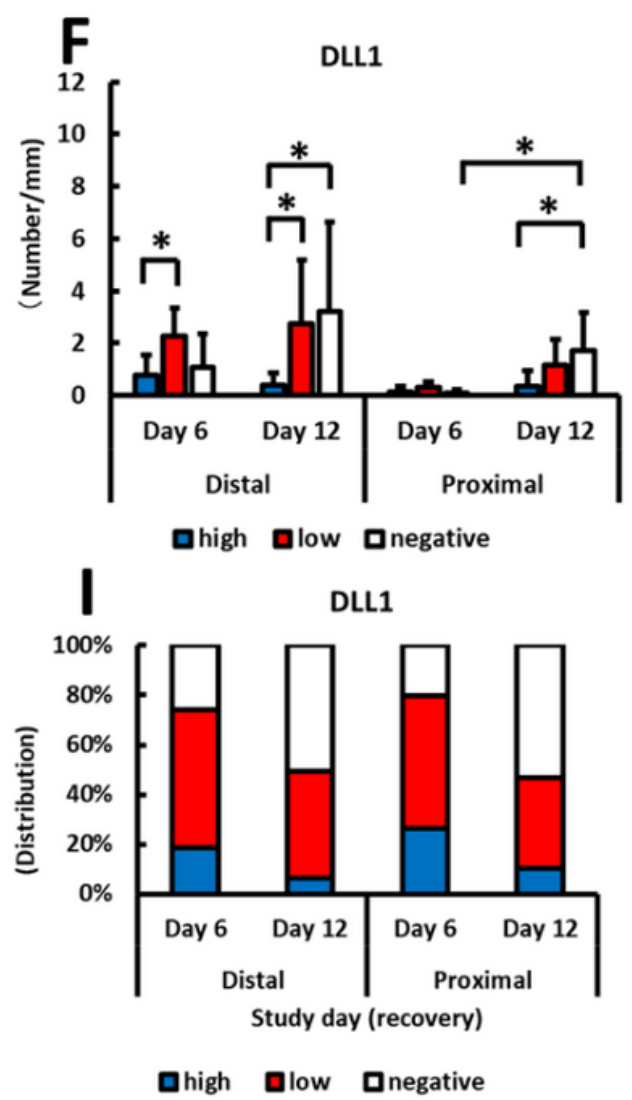

Figure 5

Coexpression analysis of stem cell and progenitor cell markers in the epithelial clumps in the lamina propria in the colorectum of mice administered 5\% DSS in drinking water for 6 days (Day 6), followed by withdrawal of DSS for 6 days (Day 12). Representative images of high and low intracytoplasmic expression of LGR5 (A), BMI1 (B), and DLL1 (C) in epithelial clumps (Red circles). Bar=10 $\mu \mathrm{m}$. The number of epithelial clumps with high and low expression levels (D-F) per millimeter and distribution pattern (G-I) of each marker in epithelial clumps in the lamina propria in the distal and proximal colorectum. (D-F) The data represent the means and standard deviations. *, **Significantly different between each time point or region ( $<<0.05$ or 0.01 , Tukey-Kramer test or Steel-Dwass test). 
A

SOX9/DLL1

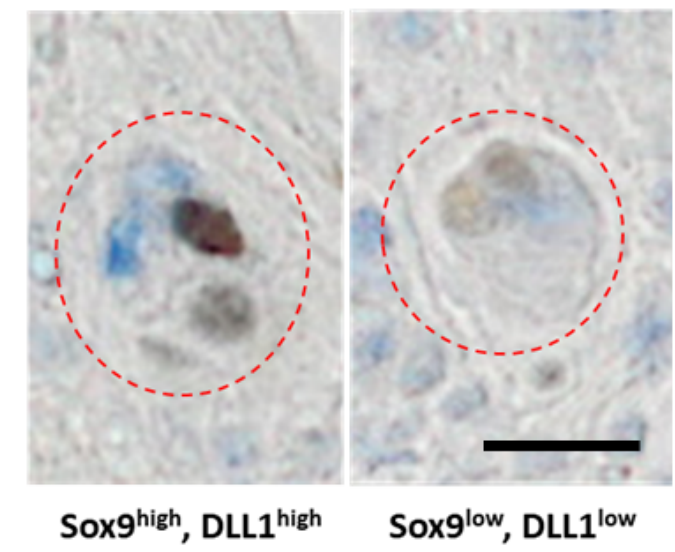

C

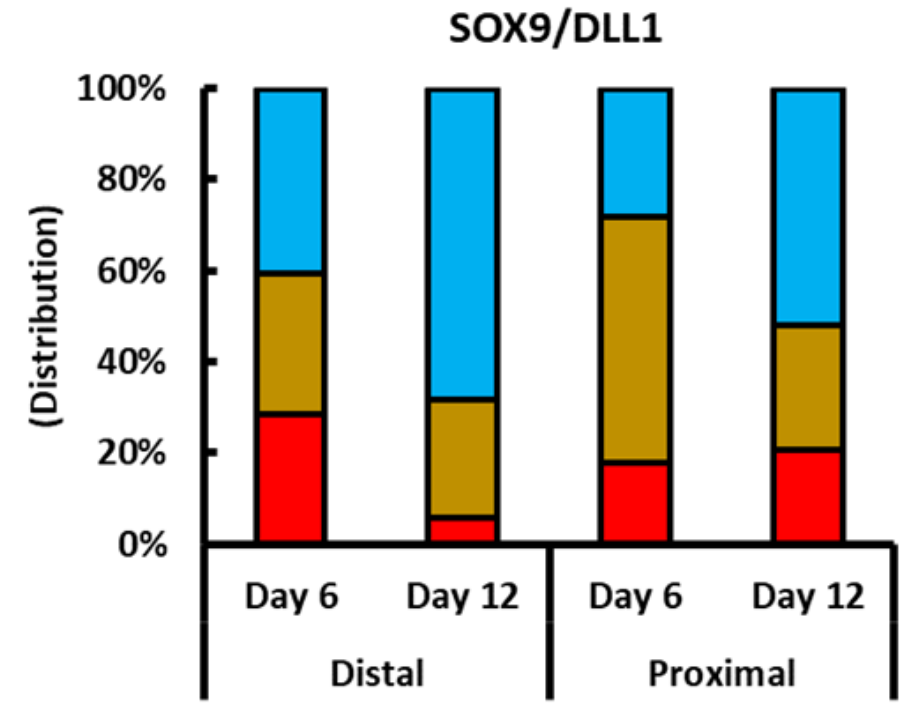

aSOX9/DLL1 口SOX9 aDLL1
B

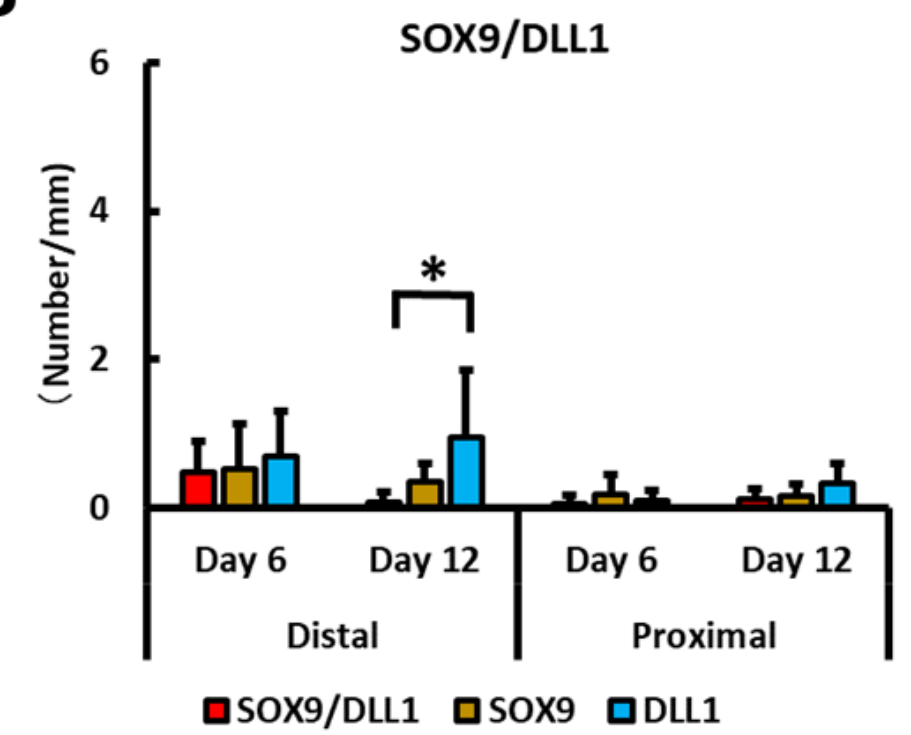

D

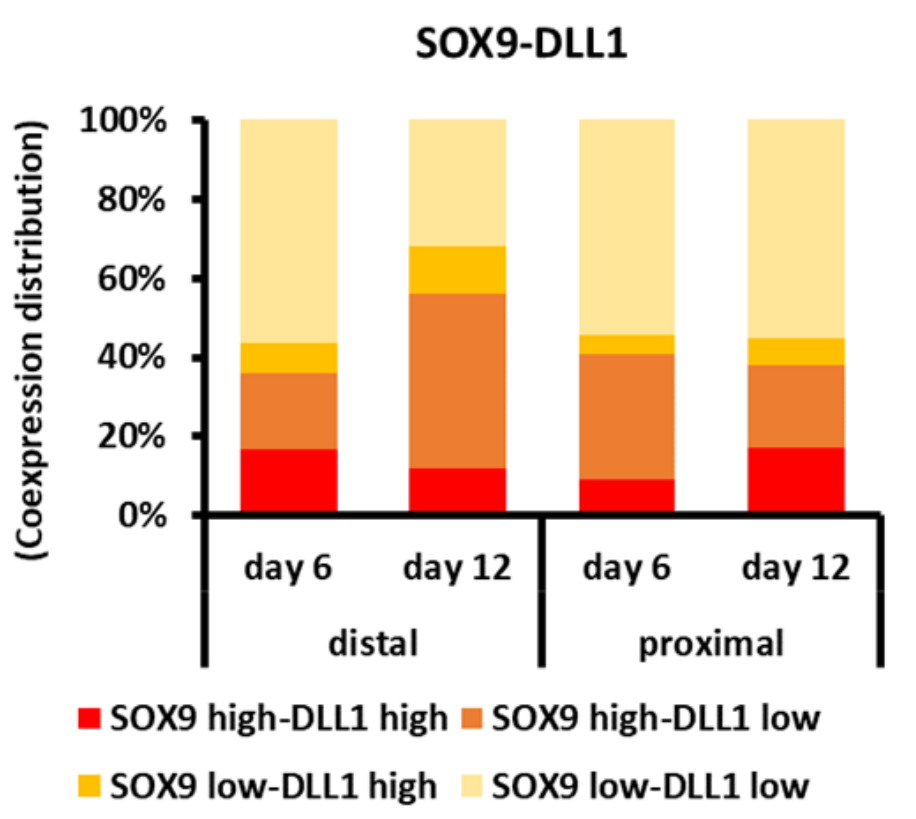

Figure 6

Coexpression analysis of SOX9 and DLL1 in the epithelial clumps in the lamina propria in the colorectum of mice administered 5\% DSS in drinking water for 6 days (Day 0), followed by withdrawal of DSS for 6 days (Day 6). (A) Representative images of high and low nuclear expression of SOX9 and intracytoplasmic expression of DLL1 in epithelial clumps (Red circles). Bar=10 $\mu \mathrm{m}$. The number of epithelial clumps with coexpression or each expression per millimeter (B) and distribution pattern (C) of each marker in the epithelial clumps. The data represent the means and standard deviations.

*Significantly different between each time point ( $p<0.05$, the Tukey-Kramer test or Steel-Dwass test). (D) Coexpression distribution of SOX9 and DLL1 at high or low expression level. 


\section{Supplementary Files}

This is a list of supplementary files associated with this preprint. Click to download.

- KobayashiSupplementallnformation.docx 